\title{
Comparison of hip joint mechanical energetics in table tennis forehand and backhand drives: a preliminary study
}

Thibault Marsan I, Philippe Rouch I, Patricia Thoreux I, 2, Rozenn Jacquet-Yquel 3 and Christophe Sauret I

I Arts et Métiers ParisTech, Institut de Biomécanique Humaine Georges Charpak, Paris, France

2 Hôpital Avicenne, Université Paris I3, Bobigny, France

3 INSEP, Pôle France de Tennis de table, Paris, France

\section{Abstract}

Hip joints are highly involved in table tennis. Some authors found both pelvic angular velocity and hip joint torques are related to the racket velocity. Others have also demonstrated how some of the best players have higher ranges of motion of the lower-limb joints. Therefore, the mechanical work generated by the playing-side-hip can be seen as indicator of the playing intensity associated with different strokes. The aim of this study was to quantify the hip joint mechanical work and power during four classical strokes. Motion capture acquisitions were performed on two international players. A biplanar radiographic acquisition was also performed to personalize the biomechanical model. Hip joint velocity and torques were calculated on the dominant side, allowing mechanical power and work to be calculated between the end of backswing and the ball impact. The highest level of mechanical work from the hip joint was found for forehand drive against backspin and forehand topspin drive with pivot. A backhand drive required the lowest hip mechanical work, and the forehand drive against topspin was found to be intermediate. The lower work required from the backhand stroke makes it suitable as a waiting stroke.

Keywords: Table Tennis, Hip Joint, Mechanical Work

Correspondence author: Thibault Marsan

E-mail: thibault.marsan@ensam.eu

Cite this article as:

Marsan, T., Rouch, P., Thoreux, P., Jacquet-Yquel, R., \& Sauret, C. (2019). Comparison of hip joint mechanical energetics in table tennis forehand and backhand drives. International Journal of Racket Sports Science, 1(2), $37-43$. 


\section{Introduction}

One of the most used stroke in table tennis is the topspin forehand, which accounts for $36 \%$ of the total shots performed during a game (Lanzoni, Di Michele, \& Merni, 2014). Previous kinematic studies on table tennis focused on evaluating the relationship between joint angles and racket velocity during topspin forehand drives (Bańkosz \& Winiarski, 2018; Iino \& Kojima, 2009; Qian, Zhang, Baker, \& Gu, 2016). Few of them focused on lower limb kinematics (Qian et al., 2016) but it was observed that the best players tend to exhibit a higher hip range of motion during topspin forehand drives than players of a lower level. Kinetic aspects of table tennis have been less studied: only one study focused on hip kinetics (Iino, 2017) in which a positive correlation was found between the horizontal velocity of the racket and both hip joint torques and pelvis angular velocities.

Energetic aspects (i.e. mechanical work and power), which are at the interface between kinematics and kinetics analysis, have been studied in other sports, such as rugby tackles (Hendricks, Karpul, \& Lambert, 2014), tennis serves (Martin et al., 2014) or badminton shots (Rasmussen, Kwan, Andersen, \& de Zee, 2010), allowing the understanding of the physical demand of specific sport associated gestures.

In table tennis, the energy flow from the trunk to the racket arm has already been investigated and this study showed that most of the energy transmitted to the racket came from the trunk, and that lower limbs and trunk muscles are supposed to generate most of the energy transferred to the racket during topspin forehand drive (Iino \& Kojima, 2011). Consequently, studying energetic aspects in table tennis can provide insights into athletes' striking performances (van der Kruk, van der Helm, Veeger, \& Schwab, 2018).

Regarding the involvement of pelvic angular velocity and hip joint torques in table tennis striking performance (Iino, 2017; Qian et al., 2016), the hip joint mechanical power and work can be seen as indicators of the playing intensity required by the different table tennis stokes. Hence, the aim of this study was to quantify the hip joint mechanical power and mechanical work during four classical table tennis strokes: topspin forehand drive on topspin incoming ball, topspin forehand drive on backspin incoming ball, topspin forehand drive with pivot, and backhand drive on topspin incoming ball. It has been hypothesized that hip mechanical work and power would be higher during forehand drive on backspin incoming ball since the player has to produce more spin.

\section{Material and methods}

\section{Participants}

Two right-handed males; both international table tennis players from France, were involved in this study, in line with the previously obtained ethical agreements (2018-A00173-52). Subjects were informed of the protocol and signed a written informed consent form before the beginning of the experiments. Subjects' characteristics were: age: 20 and 22 years; body mass: 86 and $75 \mathrm{~kg}$; and height: 1.92 and $1.87 \mathrm{~m}$, for participant 1 and 2, respectively.

\section{Data collection}

Participants were equipped with 88 reflective markers fixed on their whole body plus three on the racket (Figure 1). This allows a full-body analysis and the definition of segment coordinate systems, in line with recommendations from the International Society of Biomechanics (Wu \& Cavanagh, 1995; Wu et al., 2002, 2005). After completing their own warm up routine to get comfortable with the environment and experimental setup, the participants completed at least 5 cycles of each of the four activities: topspin forehand drive on topspin incoming ball (FH_TS), topspin forehand drive on backspin incoming ball (FH_BS), topspin forehand drive with pivot on a topspin incoming ball (i.e. a topspin forehand drive with a lateral displacement of the player) (FH_D), and a backhand drive on a topspin incoming ball (BH_TS). Participants played against each other during the acquisitions for all activities but only one player was monitored at a time. For FH_BS, the studied player received only one ball, five times in a row. Locations of the reflective markers were captured using a 15cameras optoelectronic motion capture system (Vicon ${ }^{\circledR}$ System, $@$ Oxford Metrics Inc., UK, $200 \mathrm{~Hz}$ ) 
with simultaneous recording of ground reaction forces obtained with two force plates (AMTI BP6001200, USA, $1000 \mathrm{~Hz}$ ). Right after the motion capture and without removing the markers, participants underwent a low dose biplanar radiographic acquisition (EOS, EOS Imaging) in a neutral standing posture. The biplanar radiographs were then used to perform 3D reconstructions of the spine, pelvis, femurs, tibias, fibulas and the markers, which were used to personalize the biomechanical model.

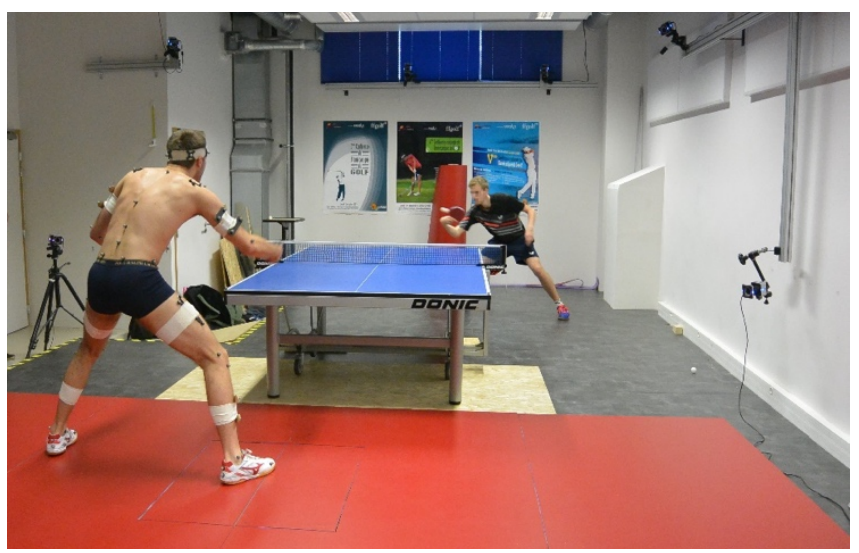

Figure 1. Photo of the research station

\section{Data processing}

Kinematics were obtained through a multibody kinematic optimization procedure ( $\mathrm{Lu} \&$ O'Connor, 1999) with a personalized full-body model (Bourgain et al., 2018) based on previously available models (Raabe \& Chaudhari, 2016; Seth, Matias, Veloso, \& Delp, 2016). The data processing was performed in OpenSim 3.3 (Delp et al., 2007). First, the multibody kinematic optimization provided joint angles. Then, joint angles were smoothed with a Butterworth filter $(5 \mathrm{~Hz}$, zero-phase, with a total order of 4$)$. Finally, force-plate data was used for computing net joint torques with the inverse dynamics tool implemented in OpenSim 3.3.

\section{Power and mechanical work computation}

Hip joint angular velocity and torque on the playing side (i.e. right side for both participants) were projected into the same orthogonal coordinate system and then multiplied to obtain the hip joint mechanical power. This mechanical power was calculated during each cycle of all four activities and normalized with respect to the body mass of the participant. The mechanical work has been calculated as the integral sum of the normalized hip power with respect to time during the strike phase. This phase was defined between the end of the backswing (i.e. the instant when the racket was at its lowest position) and the impact between the ball and the racket (here defined as the instant of maximal racket linear speed). Hip joint mechanical power and mechanical work was only calculated on the playing side (arm with the racket) because only one foot was on the force plates simultaneously. Because mechanical power can be positive or negative, we therefore distinguished the negative work, which is the time integral of the power when the power is negative (Figure 2); the positive work, which is the time integral of the mechanical power when the power is positive, and the total mechanical work, which is the sum of positive and negative works. The maximal racket speed during every stroke was also determined for the two participants.

\section{Statistical analysis}

Considering the low number of trials per activity, a non-parametric Wilcoxon-Mann-Whitney test was performed to determine if there was any significant difference $(a=0.05)$ across the activities for each variable of interest.

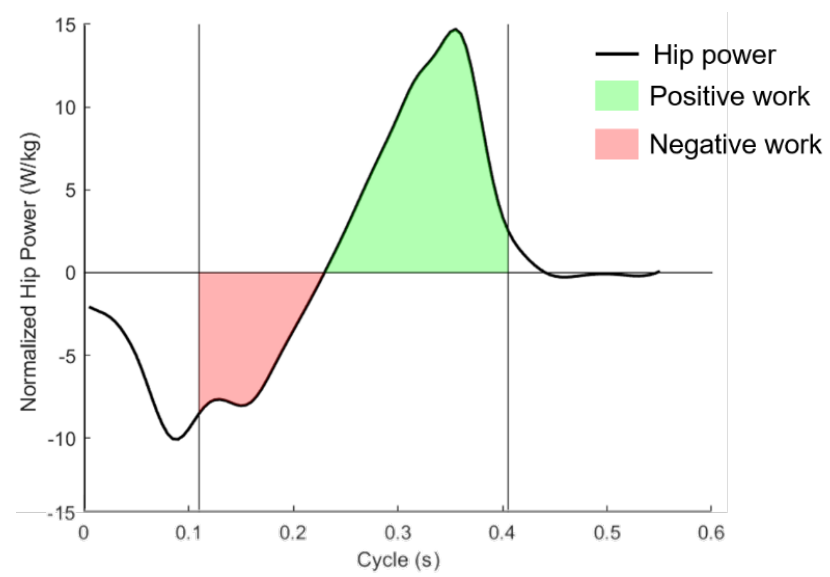

Figure 2. Example of the time course of the hip joint mechanical power (normalized with participant's mass) for one trial of one participant. The two vertical lines delimitate the strike phase. Shaded areas represent positive (green) and negative (red) works. 


\section{Results}

The maximal racket speed has been found higher in all four activities for participant 1 than for participant 2 (Table 1). For both participants, the maximal racket speed was the highest during the FH_BS. The participant 1 had significantly higher racket speed than participant 2 during all activities (Table 1).

For both participants, maximal hip joint mechanical power on the playing side was the lowest for BH_TS (Figure 3). For participant 1, the maximal power was obtained during FH_BS and FH_D followed by FH_TS. For the second participant, maximal powers were found on both FH_TS and FH_D, whereas BH_TS was the activity with lowest maximal power, preceded by FH_BS.
For both participants, the maximal hip joint mechanical power was the lowest for BH_TS. For participant 1, maximal power was obtained during FH_BS and FH_D (Figure 3).

Total mechanical work was also found the lowest for the BH_TS (Figure 4). For both participants, the highest total mechanical work was found for $\mathrm{FH}_{-} \mathrm{BS}$. Also, for both participants, the lowest negative work was found for FH_BS. The mean negative work value during the strike phase is comparable between the two participants for all activities. However, for all strokes, participant 1 exhibited wider dispersion than participant 2 for positive, negative and total works.

Table1.

Maximal racket speed during the strike (mean $\pm S D$ ) for all activities and for both participants. The * means that there were significant differences between the players.

\begin{tabular}{ccccc}
\hline & $\begin{array}{c}\text { FH_TS } \\
(\mathrm{m} / \mathrm{s})\end{array}$ & $\begin{array}{c}\text { FH_BS } \\
(\mathrm{m} / \mathrm{s})\end{array}$ & $\begin{array}{c}\text { FH_D } \\
(\mathrm{m} / \mathrm{s})\end{array}$ & $\begin{array}{c}\text { BH_TS } \\
(\mathrm{m} / \mathrm{s})\end{array}$ \\
\hline Participant 1 & $14.5 \pm 1.2$ & $19.0 \pm 0.3$ & $16.7 \pm 1.7$ & $13.3 \pm 1.4$ \\
Participant 2 & $13.2 \pm 0.4$ & $17.6 \pm 0.4$ & $16.0 \pm 0.5$ & $11.7 \pm 0.2$ \\
p-value & $0.007^{*}$ & $0.007^{*}$ & $0.007^{*}$ & $0.015^{*}$ \\
\hline
\end{tabular}
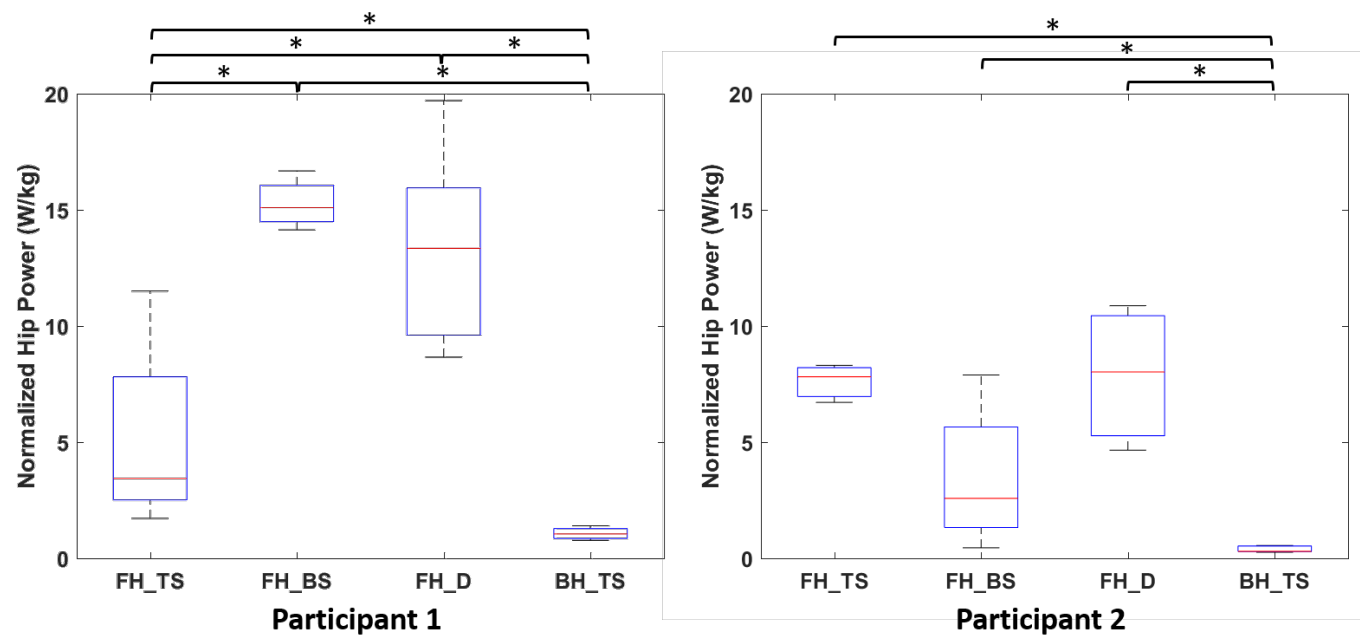

Figure 3. Boxplot of the maximal normalized hip joint mechanical power for the four activities ( 5 trials by boxplot) of each participant. The * means that there were significant differences between the activities. 

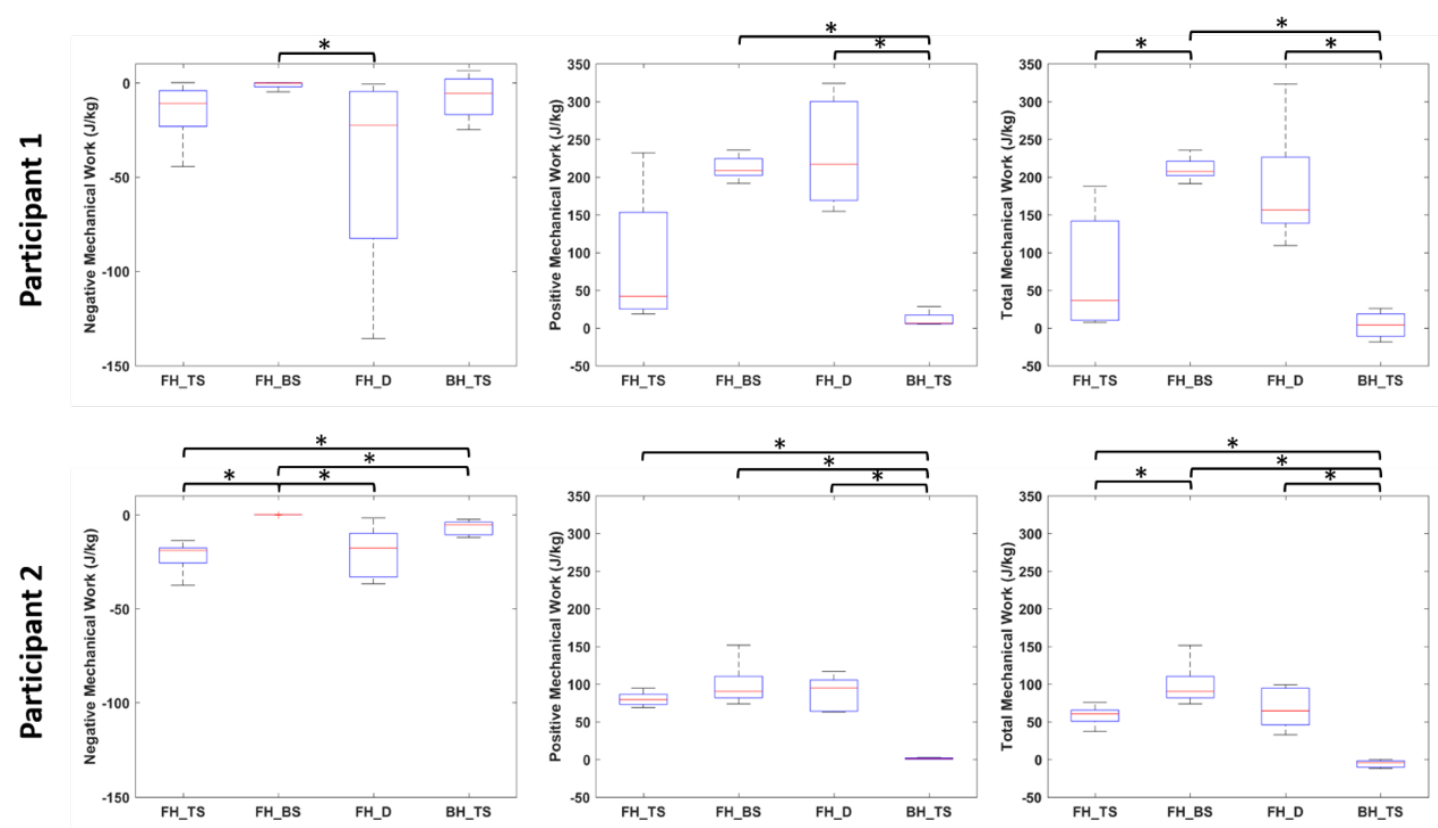

Figure 4. Boxplot of the negative, positive and total mechanical works during the strike phase for both participants during the four activities ( 5 trials by boxplot). ${ }^{*}$ indicates a significant difference between the activities.

\section{Discussion}

Values of racket speed at impact were consistent with the literature for FH_BS, which was of $18 \mathrm{~m} / \mathrm{s}$ on average (Huang et al., 2013) against balls with backspin. It is also consistent for BH_TS, which ranged between 12 and $16 \mathrm{~m} / \mathrm{s}$ (Iino \& Kojima, 2016). Furthermore, participants from this study (Iino \& Kojima, 2016) performed backhand strikes against a ball-projecting machine whilst in the present study, participants faced each other, which led to match-like situations with comparable spin and ball speed.

Since the statistical analysis has been made on only five cycles for each activity, the significant differences may not be interpreted as strong differences but should be considered as tendencies regarding the differences between participants or activities.

Results have shown that both hip joint mechanical power and mechanical work were lower during the backhand drives than during the other studied strokes. Moreover, there was very little mechanical work produced during this stroke. Indeed, the low total mechanical work is not due to compensation between positive and negative power but rather due to low absorption and production of mechanical energy. This activity is the one that requires the lowest hip physical demand during games. Consequently, players can use backhand drives as a waiting strategy because it necessitates low energy at the playing hip to perform this gesture.

The FH_BS activity required the maximal hip mechanical power for the first participant but not for the second. This means that the hypothesis cannot be validated as a generality. Nevertheless, hip joint mechanical power was higher for participant 1 than for participant 2 (Figure 3) and can be related to the higher racket speed observed for the first participant than for the second (Table 1). This result agrees with findings from previous studies investigating pelvis angular velocities and hip joint torques. However, the difference in racket speed is not high $(1.42 \mathrm{~m} / \mathrm{s}$ during FH_BS which correspond to an increase of $8 \%$ of the racket speed) whilst the difference of maximal hip power is of approximately $15 \mathrm{~W} / \mathrm{kg}$ (which corresponds to an increase of 150\%). These differences in hip joint mechanical power directly impact the mechanical work generated at the hip. Despite both participants having similar negative mechanical work during the strike, the first 
participant managed to generate more positive mechanical work which led to a higher total mechanical work. Hence participant 1 can be seen as more effective than participant 2.

The FH_D activity, which is a forehand with a lateral displacement, required higher mechanical work and power than the FH_TS activity for the first participant. This higher hip joint mechanical work and power directly impact the racket maximal velocity, which is higher for the FH_D activity than for the FH_TS one. However, even if the second participant has a higher maximal racket velocity during FH_D activity than during FH_TS activity, there was no difference in the hip mechanical power and work during these two activities. This means that the hypothesis stating that the mechanical work and power would be higher during FH_BS than FH_TS cannot be validated.

\section{Conclusion}

Based on previous knowledge about the relation between the racket velocity and both the pelvic angular velocity and the hip joint torques, this study aimed to investigate the hip mechanical energy during four classical table tennis stokes. This preliminary study showed that backhand drives can be used as a waiting strategy while conserving energy in the case of the playing side hip. On the contrary, forehand strokes against balls with backspin require high hip joint mechanical work and power to be produced. Hence, backspin strokes can be used to increase hip opponent exhaustion. Nonetheless; this can increase the exposure to opponent attack.

Through the distinction of positive and negative mechanical works, it was possible to analyze the biomechanical efficiency of the stroke between participants. This distinction allowed observing that both participants used different strategies to generate hip power. However, at this stage, this analyze was limited to the playing-side hip.

Finally, this study is a preliminary study and more subjects are needed to confirm the results. It would also be interesting to study the power flow, including that of the lower limb, during these classical table tennis strokes.

\section{References}

Bańkosz, Z., \& Winiarski, S. (2018). The evaluation of changes of angles in selected joints during topspin forehand in table tennis. Motor Control, 22(3), 314-337. https://doi.org/10.1123/ mc.2017-0057

Bourgain, M., Hybois, S., Thoreux, P., Rouillon, O., Rouch, P., \& Sauret, C. (2018). Effect of shoulder model complexity in upper-body kinematics analysis of the golf swing. Journal of Biomechanics, 75, 154-158. https://doi.org/10.1016/j.jbiomech.2018.04.025

Delp, S. L., Anderson, F. C., Arnold, A. S., Loan, P., Habib, A., John, C. T., ... Thelen, D. G. (2007). OpenSim: Open-Source Software to Create and Analyze Dynamic Simulations of Movement. IEEE Transactions on Biomedical Engineering, 54(11), 1940 1950.

Hendricks, S., Karpul, D., \& Lambert, M. (2014). Momentum and kinetic energy before the tackle in rugby union. Journal of Sports Science and Medicine, 13(3), 557-563.

Huang, H., Hsueh, Y., Chen, Y., Chang, T., Pan, K., Huang, K., \& Tsai, C.-L. (2013). The kinematics analysis of table tennis forehand and backhand drives. In XXIV Congress of the international society of biomechanics (pp. 3-6).

Iino, Y. (2017). Hip joint kinetics in the table tennis topspin forehand: relationship to racket velocity. Journal of Sports Sciences, 36(7), 1-9. https://doi.org/10.1080/02640414.2017.1344777

Iino, Y., \& Kojima, T. (2009). Kinematics of table tennis topspin forehands: Effects of performance level and ball spin. Journal of Sports Sciences, 27(12), 1311-1321. https://doi.org/10.1080/026404109032 64458

Iino, Y., \& Kojima, T. (2011). Kinetics of the upper limb during table tennis topspin forehands in advanced and intermediate players. Sports Biomechanics, 10(4), 361-377. https://doi.org/10. 1080/14763141.2011.629304

Iino, Y., \& Kojima, T. (2016). Effect of the racket mass and the rate of strokes on kinematics and kinetics in the table tennis topspin backhand. Journal 
of Sports Sciences, 34(8), 721-729. https://doi.org/10.1080/02640414.2015.1069377

Lu, T. W., \& O'Connor, J. J. (1999). Bone position estimation from skin marker co-ordinates using global optimisation with joint constraints. Journal of Biomechanics, 32(2), 129-134. https://doi.org/10. 1016/S0021-9290(98)00158-4

Malagoli Lanzoni, I., Di Michele, R., \& Merni, F. (2014). A notational analysis of shot characteristics in top-level table tennis players. European Journal of Sport Science, 14(4), 309-317. https://doi.org/10. 1080/17461391.2013.819382

Martin, C., Bideau, B., Bideau, N., Nicolas, G., Delamarche, P., \& Kulpa, R. (2014). Energy flow analysis during the tennis serve: Comparison between injured and noninjured tennis players. American Journal of Sports Medicine, 42(11), 27512760. https://doi.org/10.1177/0363546514547173

Qian, J., Zhang, Y., Baker, J. S., \& Gu, Y. (2016). Effects of performance level on lower limb kinematics during table tennis forehand loop. Acta of Bioengineering and Biomechanics, 18(3), 149-155. https://doi.org/10.5277/ABB-00492-2015-03

Raabe, M. E., \& Chaudhari, A. M. W. (2016). An investigation of jogging biomechanics using the fullbody lumbar spine model: Model development and validation. Journal of Biomechanics, 49(7), 1238-1243. https://doi.org/10.1016/j.jbiomech.2016.02.046

Rasmussen, J., Kwan, M. M. S., Andersen, M. S., \& de Zee, M. (2010). Analysis of segment energy transfer using musculoskeletal models in a high speed badminton stroke. In Proceedings of the 9th International Symposium on Computer Methods in Biomechanics and Biomedical Engineering, CMBBE2010. Cardiff University.

Seth, A., Matias, R., Veloso, A. P., \& Delp, S. L. (2016). A biomechanical model of the scapulothoracic joint to accurately capture scapular kinematics during shoulder movements. PLoS ONE, 11(1), 1-18. https://doi.org/10.1371/journal.pone. 0141028

van der Kruk, E., van der Helm, F. C. T., Veeger, H. E. J., \& Schwab, A. L. (2018). Power in sports: A literature review on the application, assumptions, and terminology of mechanical power in sport research. Journal of Biomechanics, 79, 1-14. https://doi.org/10.1016/j.jbiomech.2018.08.031

Wu, G., \& Cavanagh, P. R. (1995). ISB Recommendations in the Reporting for Standardization of Kinematic Data. Journal of Biomechanics, 28(10), 1257-1261. https://doi.org/10. 1016/0021-9290(95)00017-C

Wu, G., Siegler, S., Allard, P., Kirtley, C., Leardini, A., Rosenbaum, D., ... Stokes, I. (2002). ISB recommendation on definitions of joint coordinate system of various joints for the reporting of human joint motion-part I: ankle, hip, and spine. Journal of Biomechanics, 35(4), 543-548. https://doi.org/10. 1016/S0021-9290(01)00222-6

Wu, G., Van Der Helm, F. C. T., Veeger, H. E. J., Makhsous, M., Van Roy, P., Anglin, C., ... Buchholz, B. (2005). ISB recommendation on definitions of joint coordinate systems of various joints for the reporting of human joint motion - Part II: Shoulder, elbow, wrist and hand. Journal of Biomechanics, 38(5), 981-992. https://doi.org/10.1016/j.jbiomech.2004. 05.042 